\title{
Neonatal and pediatric thoracic ultrasonography
}

\author{
Gaetano Rea ${ }^{1} \cdot$ Marco Sperandeo $^{2} \cdot$ Marco Di Serafino $^{3}$ (1) $\cdot$ Gianfranco Vallone ${ }^{4} \cdot$ Paolo Tomà $^{5}$
}

Received: 14 September 2018 / Accepted: 7 January 2019 / Published online: 18 February 2019

(c) Società Italiana di Ultrasonologia in Medicina e Biologia (SIUMB) 2019

\begin{abstract}
Ultrasound examination of the thorax (TUS) can be quite suitable for children because their unique thoracic anatomy provides many acoustic windows into the chest. This review article covers techniques, indications, and applications of TUS in neonates, infants, and children, including common aspects and applications, like pulmonary consolidation and atelectasis, pleural effusion and pneumothorax and main neonatal pathologies such as respiratory distress syndrome (RDS) and transitory tachypnea of the newborn (TTN).
\end{abstract}

Keywords Chest $\cdot$ Ultrasound $\cdot$ Artifact $\cdot$ Lung disease

\section{Introduction}

Ultrasonography is a very powerful non-invasive diagnostic tool widely employed for examining multiple organs and systems located both superficially and deeply. This is possible thanks to a wide range of transducers that work on different frequencies and sound lengths to explore all layers of anatomical structures. Traditionally, air has been considered an 'enemy of ultrasonography'. This is because ultrasound propagation velocity in soft tissue and the parenchyma ranges between $1440 \mathrm{~m} / \mathrm{s}$ and $1590 \mathrm{~m} / \mathrm{s}$, showing the optimal propagation velocity in fluids [1]. Ultrasound propagation velocity in the air is $330 \mathrm{~m} / \mathrm{s}$, which is $2 / 3$ as fast [2]. For these reasons, pleural-lung ultrasonography has long been excluded from a consistent and complete evaluation. A good

Marco Di Serafino

marcodiserafino@hotmail.it

1 Department of Radiology, "Vincenzo Monaldi” Hospital -AORN Ospedale Dei Colli, Naples, Italy

2 Department of Internal Medicine - Interventional Ultrasound Unit, IRCCS “Casa Sollievo Della Sofferenza" Hospital, San Giovanni Rotondo, Foggia, Italy

3 Department of General and Emergency Radiology, "Antonio Cardarelli" Hospital, Antonio Cardarelli st 9, 80131 Naples, Italy

4 Department of Paediatric Radiology, "Federico II" University Hospital, Naples, Italy

5 Department of Diagnostic Imaging, "Bambino Gesù" Children Hospital, Rome, Italy example is the thoracic district-comprising of the costal skeleton and muscular, vascular, lymphatic, venous, arterial, and mediastinum structures-which faces obstruction in the propagation of mechanical waves caused by the lung. However, the presence of lymphatic and interstitial vessels in the area allows for a slight incremented velocity of ultrasound ( $440 \mathrm{~m} / \mathrm{s}$ versus $330 \mathrm{~m} / \mathrm{s})$ in relation to its diffusion in air [3].

It is important to stress that ultrasonography does not make for a thorough and complete study of the lung, as transducers cannot go through the costal skeleton; therefore, pleuropulmonary evaluation is only possible through interand trans-costal longitudinal access, both axial and sidelong; the pulmonary and pleural elements of the underneath costal skeletal structures are inaccessible and thus impossible to evaluate. It is estimated that ultrasound examination of the pleuro-pulmonary area only allows for the examination of $60-70 \%$ of the pleural surface. This means that ultrasound examination of the thorax (TUS) is useful for viewing pleural and sub-pleural diseases adherent to the pleura-a portion of the pleura that corresponds to a very small part of the entire pulmonary tissue [4]. Using CT (computed tomography), it is possible to carry out a global and complete investigation of pleural and pulmonary tissue. The pleuro-pulmonary area to the mediastinum is not explorable with ultrasound. Only masses adherent to the antero-superior mediastinal pleural are visible.

Cardiac and respiratory movements cause a reduction in diagnostic accuracy relative to the color and power Doppler-flash artifacts-obstructing a thorough study of possible vascular alterations. For all the above reasons, TUS 
cannot be a substitute for conventional radiological methods, such as chest X-ray and CT that allow the complete investigation of thoracic volume in multi-parametrical details (MDCT), which is extremely useful for an accurate diagnosis. However, the radiobiological impact-free Magnetic Resonance Imaging (MRI) employed in pediatric evaluation still show a limited resolution due to less optimal signal/ noise rapport vs acquisition time.

In contrast, TUS is a valuable integration tool for goldstandard methods. This is also thanks to the presence of sonographists in all intensive pediatric and neonatal therapy structures. Ultrasound examination has high diagnostic value when applied appropriately, and it is virtually free from the damaging biological effects caused by applying ionizing radiation [5].

Other advantages include the portability of the equipment, which allows for even bedside examination, the comfort of the patient, reputability, and reduced cost.

The training and advanced study of ultrasound techniques, thorough knowledge of human anatomy, the study of ultrasound artifacts, and ultrasonographic semiotics are all crucial tools for an ultrasonographist. This is particularly true because the availability of quality and portable ultrasound machines, often used in intensive care units (ICU), has led to the application of various techniques in the investigation of a given pathology by professionals from different backgrounds.

Furthermore, the lack of specific guidelines has caused diagnoses to be reported in unclear and inconsequent ways, this endangering patients' management. Such risks have been amplified by an increase in borderline and off-label approaches to the study of pleuropulmonary pathology. The goal of this paper is to offer an analysis of pleuro-pulmonary pathology in the neonatal age for which ultrasonography can have a detective and diagnostic role, and can support the management of young patients. Nonetheless, we acknowledge that the diagnosis and management of several main neonatal pathologies are still a work in progress.

\section{Ultrasonographic aspects and techniques}

Neonatal TUS requires micro-convex and linear multi-frequency transducers of a frequency range of 5-15 MHz [6].

Thoracic district examination is done in different positions, based on clinical conditions: supine or prone on the left and right sides. In a few cases (e.g., the patient is in an incubator or depends on a ventilator machine; clinostatic position), the only available positions are supine or minimal sideway exploration. The examination of the area is divided by regular anatomical landmarks according to the well-known lines running back to front: the superclavear window, supra-sternal window, subxiphoidal anatomical space, trans-diaphragm region, parasternal region, medioclavear region, anterior, medial and posterior axillar space, and the inter-scapular and paravertebral areas [7].

An ultrasound examination of the chest is performed through axial, longitudinal, oblique, and sidelong scans modulated over intra-costal anatomical structures. Frequency modulation, available on manual multi-frequency transducers, can be useful to isolate any possible areas of interest: pleura found superficially with increased frequencies employable in the examination of the pneumothorax, deeply situated pathological lungs found with reduced frequency to allow a better evaluation of the consolidations, atelectasis caudal extension, etc.

Incomplete thoracic ossification allows for a better analysis of portions of the anterior thorax and mediastinum in newborns than in the adults due to sternal and osteochondral cartilage. No ultrasonographic information can be found in the back-scapular district and in the middle/lower mediastinal area.

The echotomographic investigation is complemented by both motion and static pictures, and real-time imaging, which allow evaluating inhaling and exhaling movements $[7,8]$. Such methods provide double information which, combined with clinical exams and a correct interpretation of the standard sonographic data or similar approaches, can help the diagnosis. Still, it is important to note that there are several factors that influence a patient's clinical information: pulmonary air and physical principles, for instance, can interfere with scanning, as they are mainly gaseous structures. This means that forcing a sonographic semiotic to correspond to clinical data is counterproductive and dangerous, as it jeopardizes both the work of the pathologist, who may come to wrong conclusions, and the patient, who may possibly suffer from a misdiagnosis.

Artifacts such as casual variations caused by ultrasound physics are, therefore, no basis for a correct diagnosis, as they can be mistaken for actual information relative to the morphology of the lung $[2,7]$. Furthermore, because the standardized hardware wave calibration available on most commercial ectomographers is $1540 \mathrm{~m} / \mathrm{s}$, it is barely effective to establish a diagnosis of pulmonary pathologies when the wave is reduced to a quarter of its velocity of transmission in the pulmonary area (about $440 \mathrm{~m} / \mathrm{s}$ ) $[2,7]$.

Advocates for non-homologated thoracic ultrasound and its diagnostic value are still researching different possible applications of ultrasound, which will only be recognized once they can prove their effectiveness, reproducibility, and easy applicability.

In those cases where the artifacts are generated by lowfrequency transducers, it is necessary to increase their frequency to a medium or high level, combining them with compound imaging and speckle reduction. Doing 
this reduces any artifacts, though such elements might be rejected by most clinical physicians, who base their management and ecotomographic observations of certain anatomical-physiological-pathological features on the visibility, number, origin, and position of such artifacts [7].

When a transducer is in touch with the thoracic wall, we can observe the following anatomical structures (Fig. 1):

Chest wall: composed of dermic and subdermic walls and adipose tissue (all medium to low echogenicity structures); rib: (hyperechoic with posterior acoustic vacuum due to the complete absorption of the ultrasonographic band into the bone); intercostal muscular planes: composed of intercostal and muscular bands (medium echogenicity); hyperechoic pleural line: (summation of parietal pleura, pleural space, and visceral pleura) represents the artifactual differential net between the tissues of different acoustic impedance, such as soft subcutaneous tissues and air underneath the lung. This line has a substantially increased volume about 150-250 microns, higher than the regular dimensional anatomical value because of the physical characteristics of its relative ultrasound $[1,2]$. Furthermore, it varies depending on the frequency of the transducer as well as the patient's body mass index (BMI), as the latter causes increased acoustic impedance, which in turns leads to an artifactual thickening of the hyperechoic pleural line [2].

The hyperechoic line, in subjects not affected by respiratory pathology, is motile and in sync with respiratory activity. Such movements are linked to pleural sliding. The 'gliding' or 'sliding sign' can indicate the absence of pneumothorax with an accuracy close to $100 \%$. Such a conclusion is further proven when the ring-down artifacts (B-lines) are found along with the comet tails, which move in sync with the child's breathing $[9,10]$.

Through time motion (TM mode), it is usually easy to observe any region of interest (ROI) along the hyperechoic pleural line constituted by a typical linear structure. The motile and fixed parts of such structures give two different, distinguishable signals. This sign, known as the sea shore sign, indicates pleural movement and natural sliding, further proving the absence of a pneumothorax.

When no pleuro-pulmonary pathology is found, it is virtually impossible to investigate the lungs beyond the immediate sub-pleural portion. This is due to the sub-total reflective artifacts, as about $99 \%$ of the ultrasound wave is reflected in healthy, normally aerated lungs, which have very limited diffraction and deep diffusion of waves, preventing any further examination of the lung [11].

Due to the physical characteristics of body structures with different densities and anatomical numbers, acoustic impedance of the tissues presents different echogenic responses, some of which are simple acoustic aberrations needed by the sonographer for easy recognition to avoid serious mistakes in the diagnosis (artifacts) [2, 7]. If such artifacts are mistakenly taken as a sign of pathology instead of being interpreted as simple conditions sometimes present in healthy subjects, it is possible to come to the wrong semantic conclusions, thus affecting the management of the patient. A deep knowledge of such artifacts allows for correct management and good interpretation of ultrasonographic pictures that would be otherwise difficult to understand.

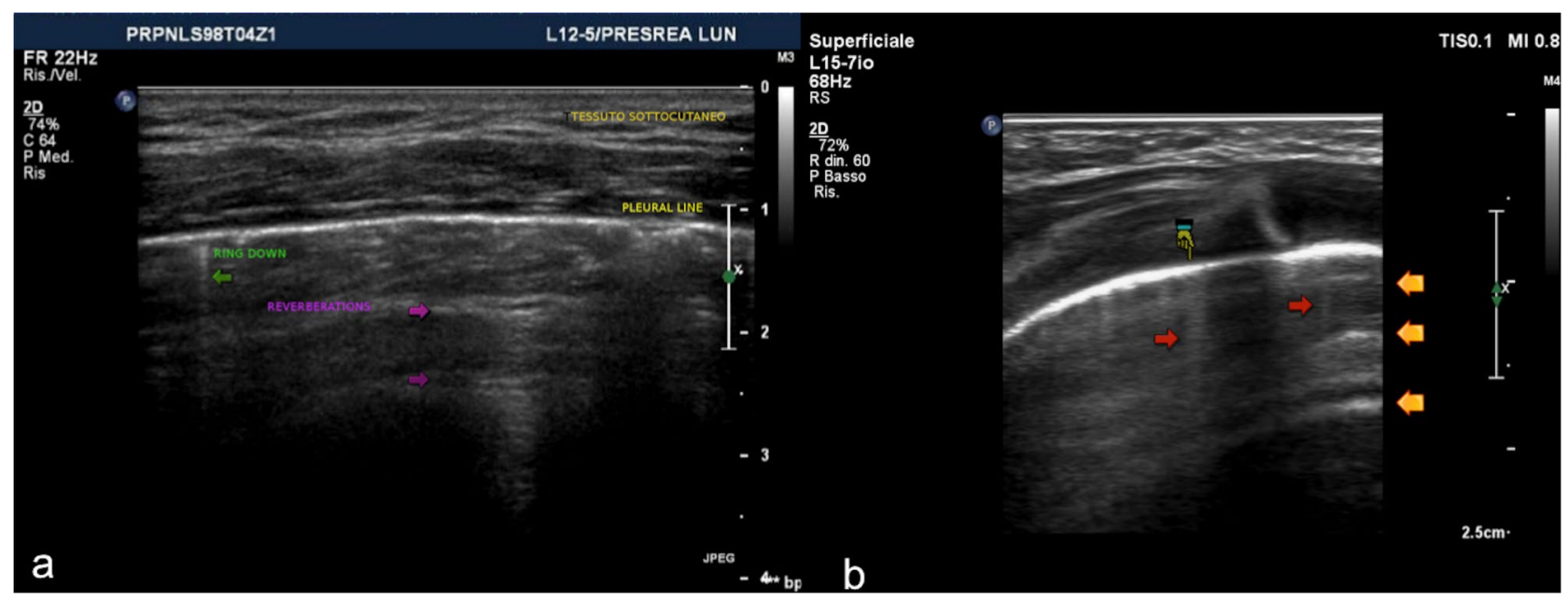

Fig. 1 Chest ultrasound examination performed with a high-frequency linear transducer (15-7 MHz) and pulmonary setting in an adult (a) and healthy newborn (b). In $\mathbf{b}$, there is a better anatomical definition of the pleural reflection (hand index finger), with evi- dence of multiple horizontal reverberation artifacts or A-lines (orange arrows) with some ring-down or B-lines (red arrows) intersecting the above A-lines 


\section{Thoracic ultrasonographic artifacts}

'Horizontal reverberations': also known as A-lines, these are the result of repeated reflections of the linear hyperechoic structure caudally to the pleural hyperechoic line [12]. Depending on the power of the transducers employed, such lines tend to fade until they disappear in deeper areas towards the caudal portion of the visual field.

They are the reflection of those ultrasound components that are not arrested, which are then fully reflected beyond the hyperechoic pleural line while keeping their acoustic power thanks to their minimal diffusion and diffraction through the air naturally present in the lung. Such artifacts are equidistant and indicate a normally aerated lung (Fig. 2).

'Longitudinal/vertical reverberations': also known as B-lines or ring-down artifacts, these originate from the pleural hyperechoic line, running like laser rays for several centimeters along the caudal line $[11,13]$. They move to the respiratory movements and are expressions of different acoustic impedance found between the film liquid components normally found in the interstitial sub-pleural portion and gaseous components found in the conductive bronchiolar and acinar alveolar portions $[11,12]$. They indicate no specific pathologies, as they are found in healthy conditions. With higher numbers, though, B-lines can be shown in some pathologies, such as sub-pleural lymphangitis, pleural effusion, even if minimal, recognized bronchial asthma, alveolar interstitial edema, congenital cardiopathologies such as IAD (inter-atrial defect) and IVD (interventricular defect), pulmonary capillary hemangiomatosis $(\mathrm{PCH})$ and alveolar/bronchial infections. For such reasons, any increase in this artifact calls for further level II clinical and diagnostic investigation, all while being aware of the extreme specificity of this sign (Fig. 3).

Soldati et al. emphasize that B-lines are signals due to pathological modifications (as interstitial lung disease) or to functional states (as deflation) that alter the volume and arrangement of the alveoli in the plane immediately below the pleura [14]. The common denominator that unites the diseases showing B-lines is the increase in the full-to-empty ratio of lung tissue immediately beneath the pleura and the spatial distribution of the residual air. B-lines are typical in the incompletely expanded posterior basal segments of normal infants. They correspond to the physiological ground glass at CT. Soldati proposes the definition "hyper-dense non-consolidative" states of the lung [14].

"Comet tail artifacts": these have been erroneously used interchangeably with B-lines, but in fact, the two artifacts present very different physical connotations and ultrasonographic characteristics. They are extremely reduced in size (3-5 mm) [11, 15]. Unlike B-lines, they immediately appear caudally to the hyperechoic pleural line, showing triangular morphology and a caudal apex. They show different acoustic impedance between tissues of different densities and anatomical number (soft tissue/metals;
Fig. 2 Chest ultrasound examination performed with a highfrequency linear transducer (15-7 MHz) and pulmonary setting in a healthy newborn: the horizontal reverberation artifact, or A-line, in a normal lung surface (green arrow) is parallel to the hyperechoic "pleural line", which is equidistant ( $\left.\begin{array}{llll}1 & 2 & 3 & 4\end{array}\right)$

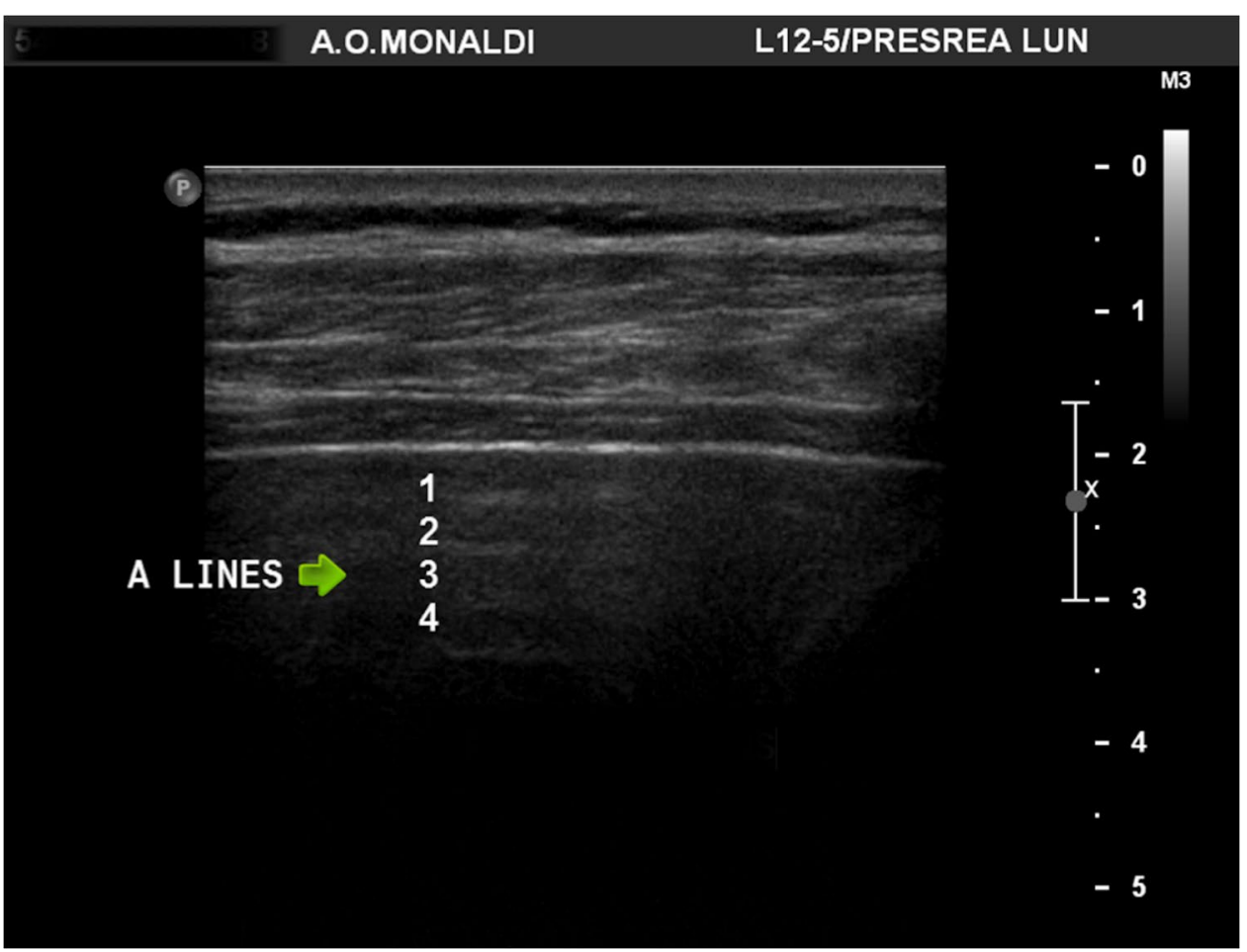


Fig. 3 Chest ultrasound examination performed with a highfrequency linear transducer (15-7 MHz) and pulmonary setting: multiple hyperechogenic linear structures or ringdown artifacts come from the hyperechoic pleural line (white arrow) progress caudally for several $\mathrm{cm}$ (orange arrows)

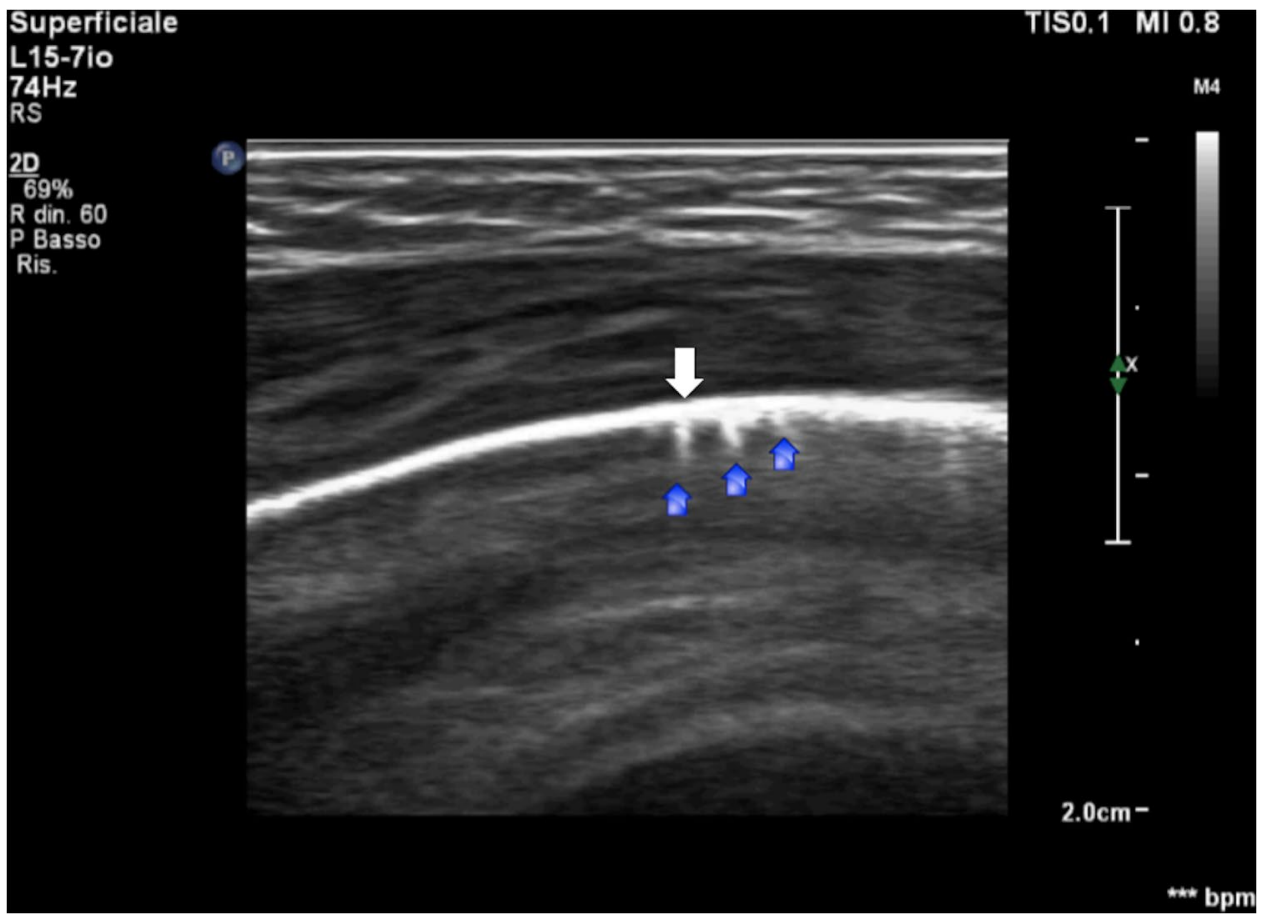

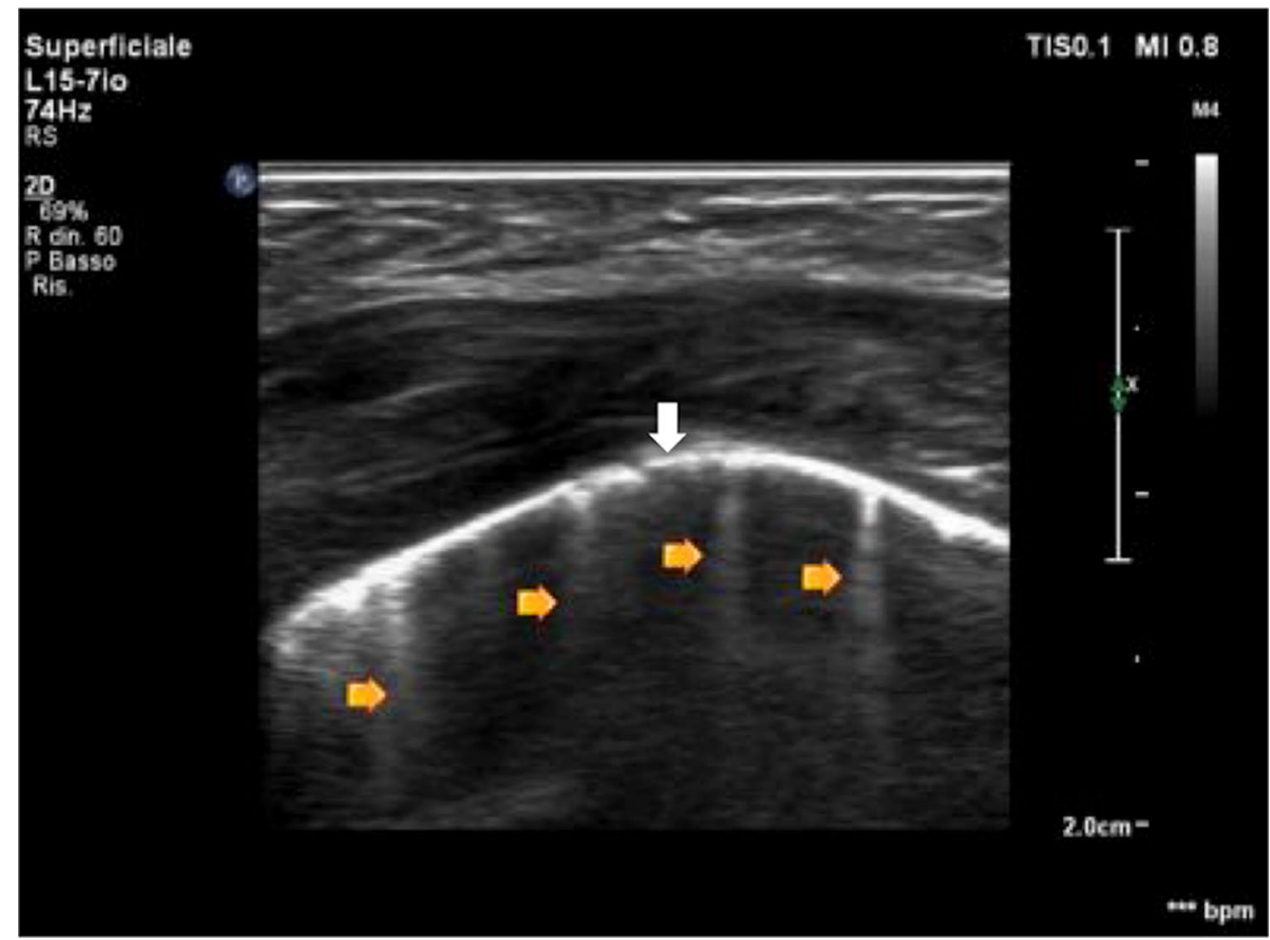

Fig. 4 Chest ultrasound examination performed with a highfrequency linear transducer $(15-7 \mathrm{MHz})$ and pulmonary setting: small linear hyperechogenic artifacts come from the "pleural line" (white arrow) and progress caudally for a few $\mathrm{mm}$ as a comet tail (blue arrows) bilis/cholesterol; soft tissue/gas). Multiple reverberations between the posterior and anterior walls of the originating structure are also typical. Comet tails never reach the casual distal portion of the echotomographic screen and move in sync with respiratory movements (Fig. 4).

\section{Validated contexts}

\section{Pleural effusion}

TUS has proven to be extremely useful for detecting pleural effusions [16]. The intrinsecal physical characteristics 
of sound waves are in fact a great tool for diagnosing even minimal effusions in the pleural space (about $<25 \mathrm{ml} \mathrm{cc}$ ). This method is, therefore, more effective than a chest X-ray, which under optimal conditions and projections is still not as effective as ultrasonography. Chest X-ray on both projections cannot detect an obliteration of the costophrenic sinus below 150-200 cc (Fig. 5a, b) [17].

Ultrasonography is an effective tool employed during serial follow-up, as it is harmless to the patient during pleural evacuation procedures when monitoring any reduction or restitution. One of the most relevant advantages of US guidance is its real-time needle visualization during the procedure [18]. The support of ultrasonography during the draining of the effusion also helps in terms of safety, amazingly reducing the risk of complications.

Scans are also useful in evaluating the neighboring lung and in detecting possible atelectasis or intrapulmonary alterations/inhomogeneity.

However, ultrasonography is not useful in the differential diagnosis between cavitary necrosis and air presence in the contexts of pneumonia, atelectasis, or cancer, though its utility in the management of pleural effusion is still valuable.

\section{Pulmonary consolidation}

In the clinical context, the sonographic examination is combined with X-ray for the management of pneumonia or atelectasis (when visible at the pleural level).

Pulmonary consolidations caused by obstruction of the alveolar compartment due to flogosis show medium to low echogenicity and irregular margins (Fig. 6), as well as internal hyperechoic spots. The latter may be due to the air bronchogram.

Major consolidations (multi-segmented or lobar) are quite rare in children until eight years, because the collateral ventilation system is not highly developed before that age [19, 20]. Nonetheless, the presence of bronchogram (either air or fluid related; ultrasonography is not able to say with certainty) is not specific of benign pathologies. It can in fact also be found in neoplastic alterations. Moreover, special emphasis is placed on the echographic result of the air bronchogram: the term air bronchogram is inappropriately used because there is no clear evidence in the literature that the hyperechoic spots and/or lines inside consolidations correlate to the $\mathrm{CT}$ imaging of a real air bronchogram $[16,21$, 22]. Furthermore, hyperechoic spots can be due to fibrotic tissue or calcification, and can even be linked to vascular/ lymphatic interfacing or the different acoustic impedance of the interstitial compartment.

For these reasons, we can only rely on CT for the multidimensional evaluation of air or fluid bronchograms in its structures, separating from the center to the periphery. Intraconsolidated or intra-atelectasis color Doppler evaluation is affected by artifacts related to cardiac and respiratory movements (flash artifacts), thus making study of the vascular signals ineffective [23].

Young children showing such symptoms are usually affected by viruses; therefore, tropism, bronchostenosis, bronchial edema, and endobronchial mucus plugging may be present, all of which contribute to 'air trapping' and microatelectasis, leading to hyperinflation.

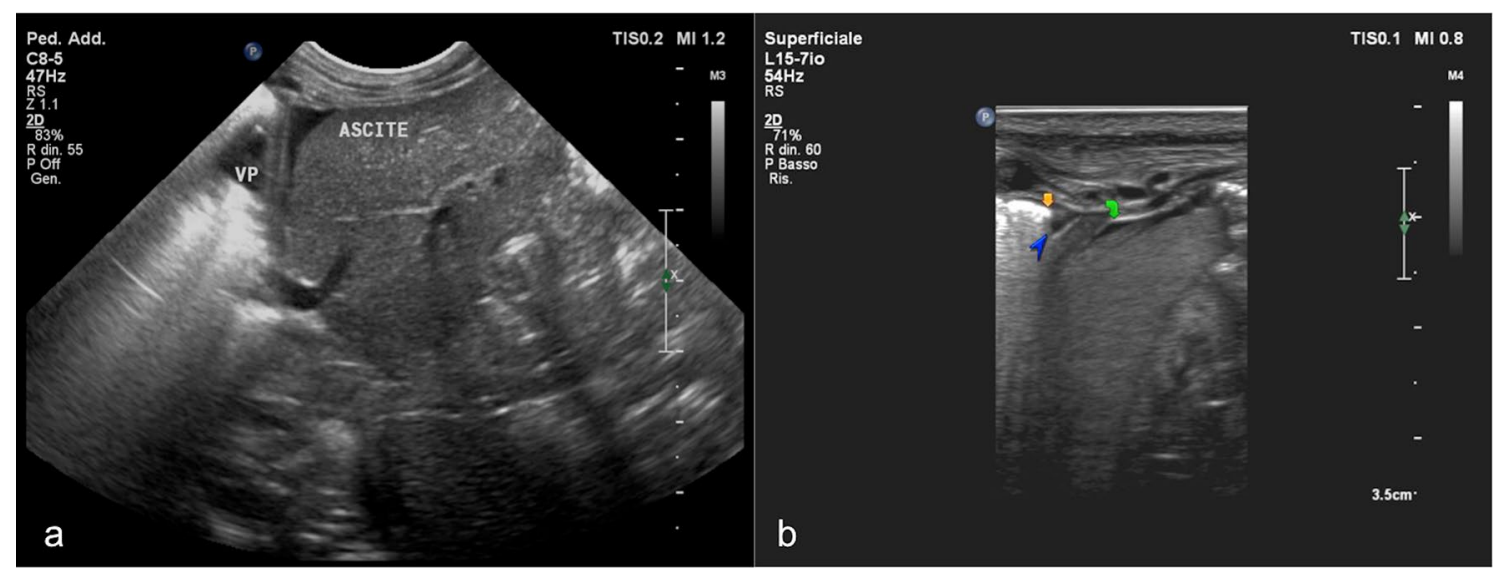

Fig. 5 Epigastric longitudinal scan with a micro-convex transducer $(8-5 \mathrm{MHz})$ and pulmonary setting, a clear detection of the hepatic left lobe with visibility of the diaphragmatic interface (white arrow) that separates the thoracic compartment with the minimal fluid component of pleural effusion (VP) and a few sub-diaphragmatic perihepatic fluid components referable to ascites. Epigastric longitudinal scan with a high-frequency linear transducer $(15-7 \mathrm{MHz})$ and pulmo- nary setting, b clear detection of the hepatic left lobe with an excellent view of the diaphragmatic interface that separates the thoracic compartment with minimal fluid components of pleural effusion (blue arrowhead), pulmonary air, outlined by the presence of the pleural line in the extreme front costo-diaphragmatic recess (orange arrow), and few sub-diaphragmatic peri-hepatic fluid components referable to ascites (green arrow) 
Fig. 6 Chest ultrasound examination performed with a highfrequency linear transducer (15-7 MHz) and pulmonary setting: small-sized bronchopneumonia (calliper) with blurring of the margins and subtle and irregular hypoechoic appearance that reaches the pleural line, deleting part of the normal pleural hyperechogenicity

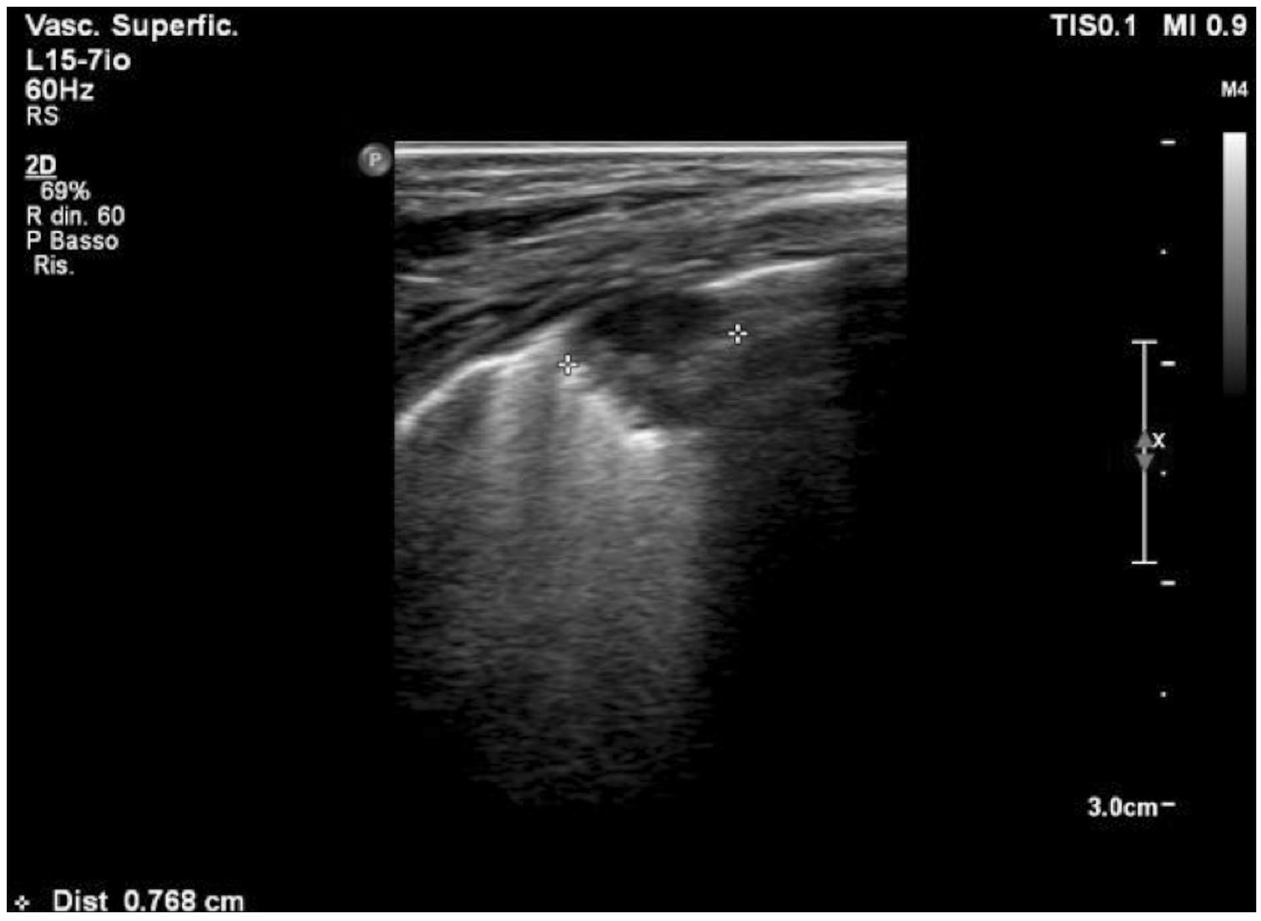

For this reason, an ultrasound of the chest following a clinical radiological diagnosis of bronchiolitis often turns out to be specific and lacks evident alterations below the pleural line beside ring-downs (Fig. 7) and the focal area of sub-pleural hypoechoic atelectasis.

Air trapping (often combined with acute inflammation) and atelectasis, with insufficient interfacing of the cardiac outline, are often key elements of a standard radiological examination [24]. Special care should be taken not to misread signs of atelectasis and confuse them for consolidations.

\section{Pulmonary atelectasis}

Such alterations can be complications of neonatal pathologies, such as respiratory distress syndrome, pneumonia, and mucous obstructions. A major sign of atelectasis is the
Fig. 7 Chest ultrasound examination performed with a highfrequency linear transducer (15-7 MHz) and pulmonary setting in patient with clinical and radiological regression (15 days away) of bronchiolitis virus infections: multiple ring-down artifacts (white arrows) on the middle and posterior axillary right line scans

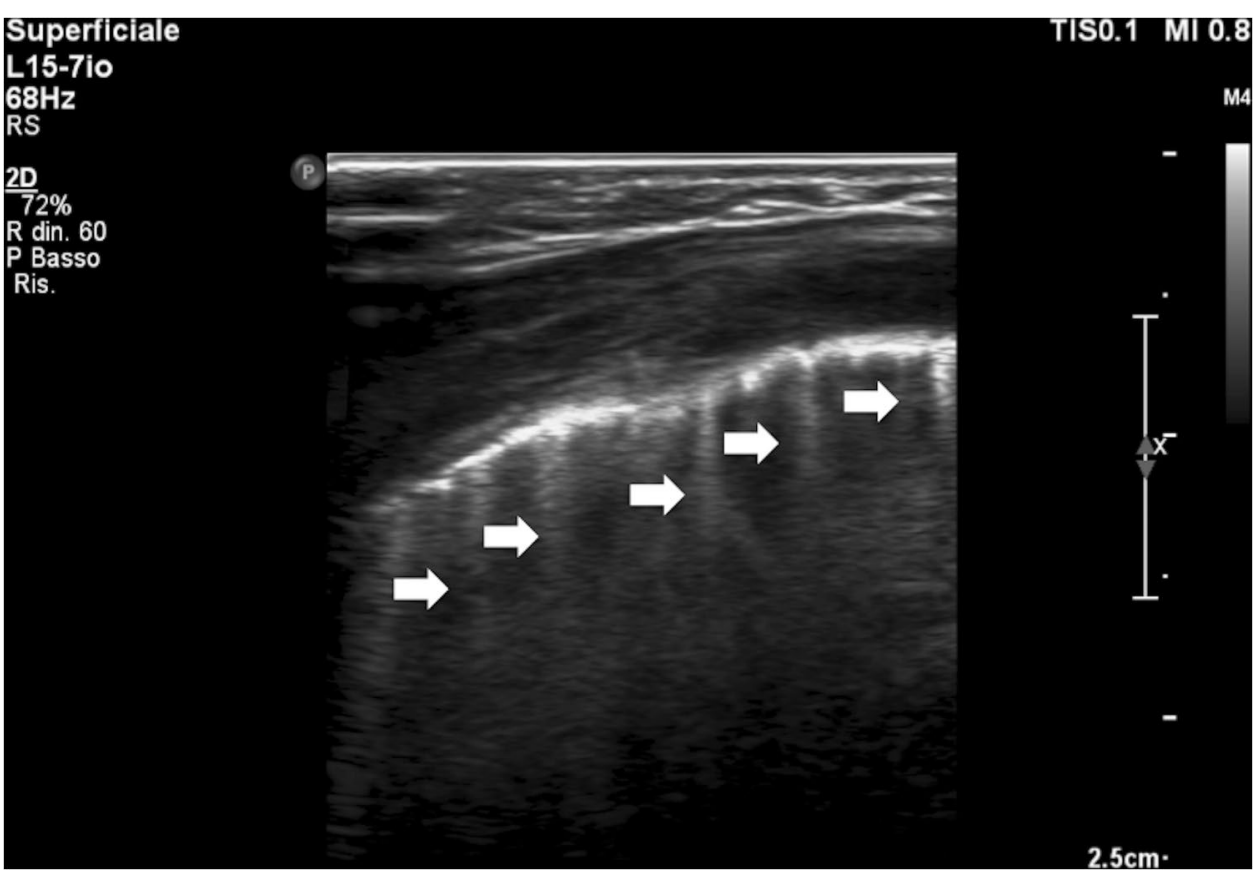


alteration of medium-to low-intensity echogenicity with more-or-less defined margins and the possible presence of hyperechoic/hypo-anechoic spots [24]. Such signs, in correlation with data collected during chest X-ray or CT examination, can lead to a picture of air/fluid bronchograms (Fig. 8). Irregularities in the pleural line can also be discovered along with the disappearance of the ring-down artifacts or increment/confluence of B-lines circumscribing the atelectasis, which causes "blurring of the atelectasis margins" [13]. The differential diagnosis between atelectasis and consolidation is the partial/total reduction of pulmonary content in the atelectatic segment of the lobe or whole lung. According to Riccabona et al., TUS cannot differentiate between atelectasis and bronchial pneumonia [6]. It is, therefore, necessary to follow-up with CT to confirm any results through a scan and possibly adjust therapeutic management accordingly.

\section{Pneumothorax}

An ultrasound examination is very useful in the detection of pneumothorax when lung sliding or gliding signs are definitely present or excluded. For this reason, US is very accurate at excluding the presence of pneumothorax unless AP chest X-ray and orthostatism projections are present, the latter being virtually impossible in neonatal intensive therapy.

Furthermore, the typical 'lung point' determines if the presence of pneumothorax is easily detectable on a scan, showing a transition from regular lung sliding to no lung sliding $[25,26]$. In those cases where lung sliding is not detectable on a scan, pneumothorax cannot be diagnosed with certainty, as the absence of lung sliding can also be a symptom of other conditions, such as RDS (respiratory distress syndrome) or reduced respiratory excursions in patients affected by acute abdomen, phrenic nerve alterations, respiratory compliance reduction in the chest apex, in obese subjects, or remarkable reduction of gliding signs in the apical due to pulmonary ligaments causing a marked fixation that strongly limits lung sliding.

\section{Main neonatal pathologies}

\section{RDS (respiratory distress syndrome) and TTN (transitory tachypnea of the newborn)}

The literature of the past decade stresses the importance of ultrasound examination in the management of some neonatal pathologies, such as RDS and TTN.

From a merely anatomical and physiological point of view, the scarce production of surfactant in premature neonates and the relative reduction of type II pneumocytes in charge of providing the substance preventing alveolar collapse cause pathological alterations with an increase of liquid components in the interstitial compartment due to capillary permeability, alveolar over-distension, and alveolar collapse (leading to a high number of ring-downs in the confluence) (Fig. 9) [27].
Fig. 8 Chest ultrasound examination performed with a highfrequency linear transducer (15-7 MHz) and pulmonary setting in patients with Down syndrome, inter-atrial defects, and perinatal asphyxia: geometric areas with irregular margins and some hyperechoic spots in the context (blue arrows) on the posterior axillary anatomical right site scans

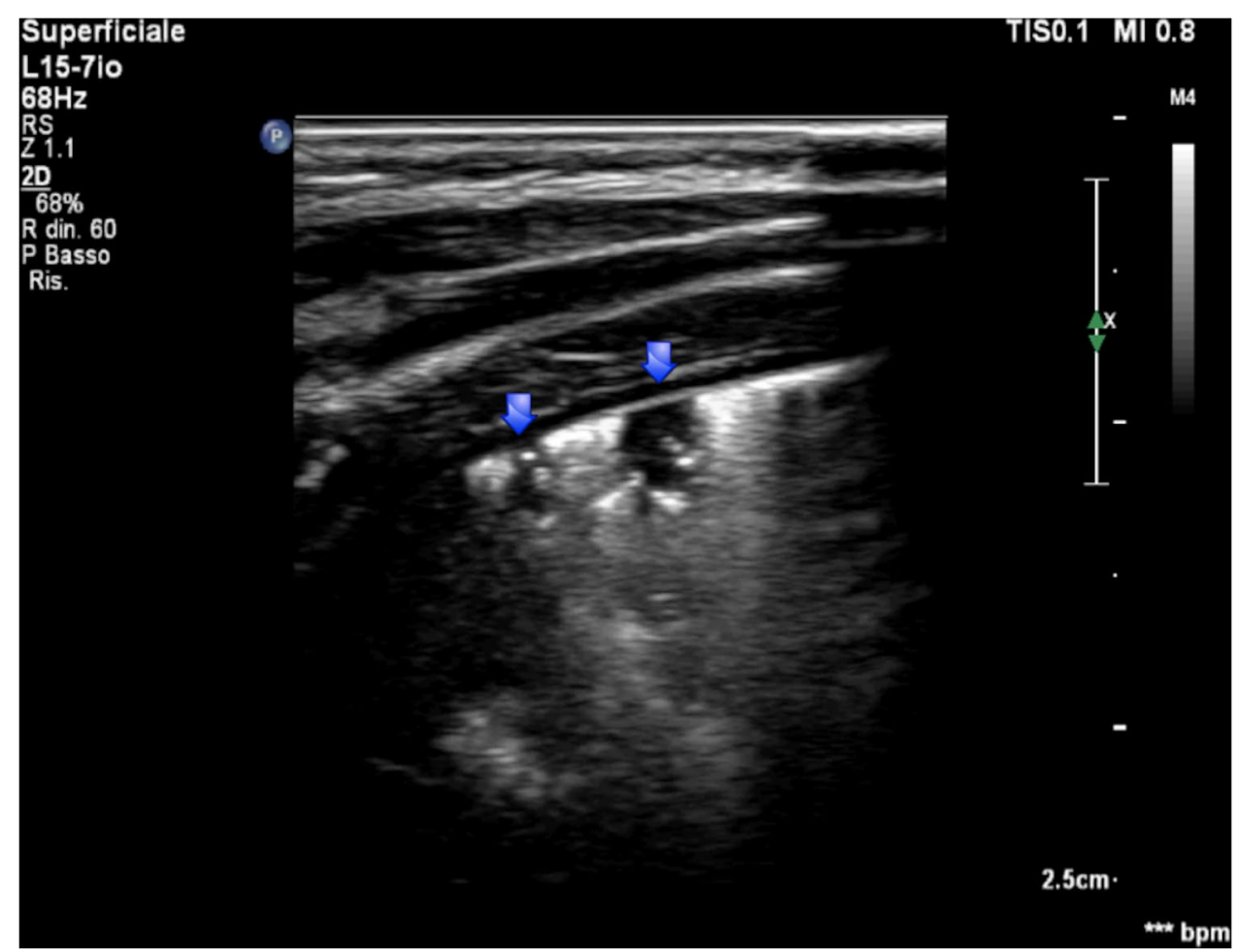


Fig. 9 Chest ultrasound examination performed with a highfrequency linear transducer (15-7 MHz) and pulmonary setting in a distressed newborn: a high number of ring-downs in confluence

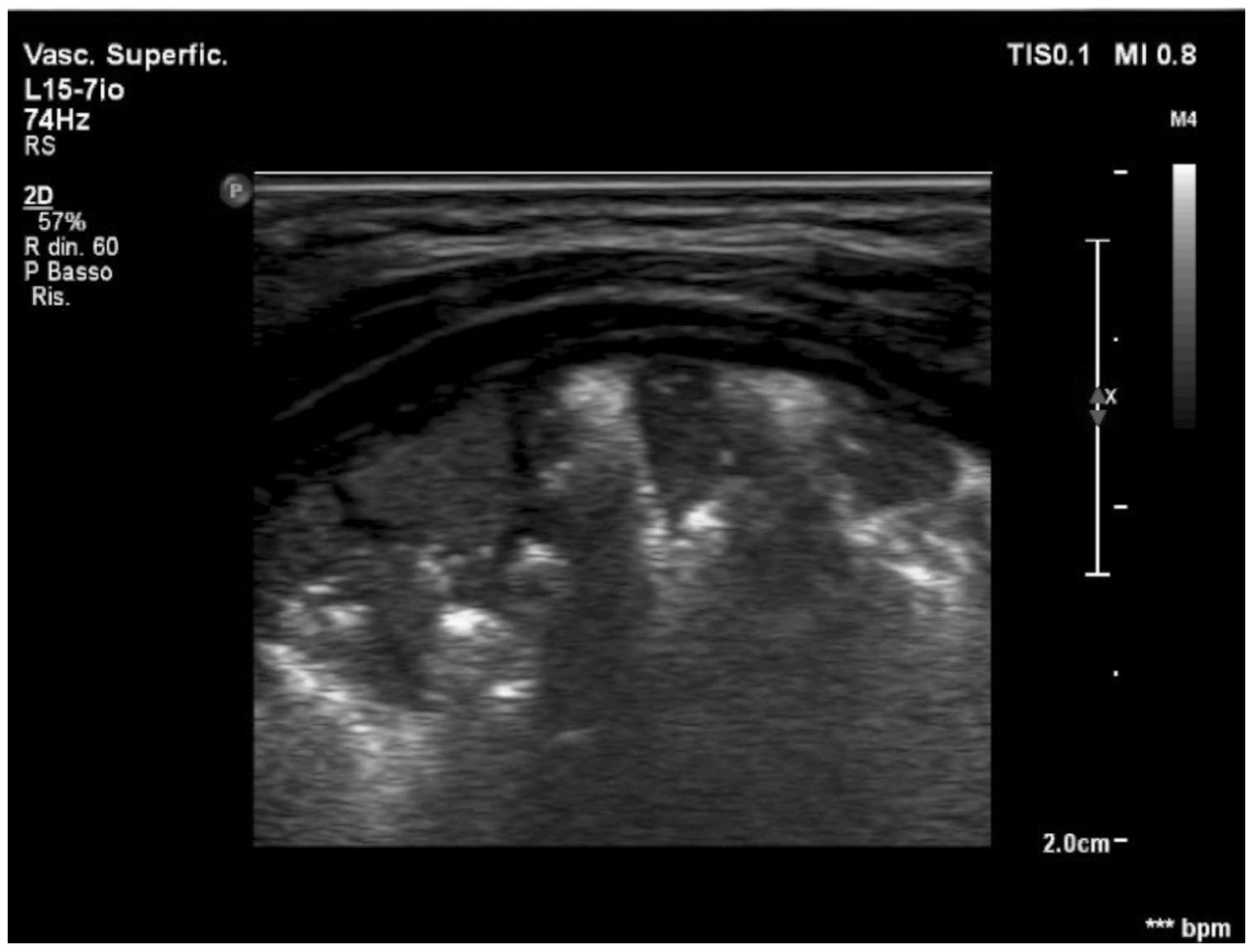

Such a report is in line with the clear hydro-gaseous imbalance induced by a pathological condition. Nevertheless, what happens in the immediate anatomical sub-pleural space cannot be projected on the entirety of the lung, as artifacts such as the pleural hyperechoic line cannot be evaluated in the same way as the lower lung, which needs instead to be monitored and managed through consolidated imaging on a chest X-ray. This is necessary, as the lower lung is obscured by both the physical sub-total reflection of the ultrasound wave and a combination of artifacts.

In at-term neonates, however, a common disorder is delayed pulmonary clearance (at variable timing), leading to the persistence of fetal liquid in the lungs [15, 27].

The timely clearance of such liquid is crucial for the transition from fetal respiration to normal air breathing. Most cases regress spontaneously and quickly, and do not require more than two radiograms of the chest. Even so, such events cause a congestion of the lymphatic system with an increase in liquid components in the interstitial compartment. Due to hydro-gaseous imbalance, such an inhibition leads to increased ring-downs with a more-or-less compact structure and "undefined sub-pleural blurring showing a feature of 'false' white lung appearance" [28].

This definition is not accurate, as a pulmonary scan can give a reliable feature next to the pleural line. In the absence of a physical means through which an ultrasound wave can easily travel (fluid, atelectasis/constitutive tissue, etc.) and when air is found in the lungs, the ultrasound wave will in fact be almost entirely reflected. This is why the lung can never be thoroughly evaluated on a scan. Therefore, due to the limits of current ultrasound machines, speaking of 'white lung' is not realistic.

\section{Conclusion}

In conclusion, it is crucial to emphasize the danger of such artifacts in the management and diagnosis of certain pathologies outside of a validated context. This is true despite the ever-growing enthusiasm for standardizing a method that is easily accessible and handy, especially to non-specialized practitioners.

\section{Compliance with ethical standards}

Conflict of interest The authors declare that they have no conflict of interest.

Informed consent All procedures followed were in accordance with the ethical standards of the responsible committee on human experimentation (institutional and national) and with the Helsinki Declaration of 1975, and its late amendments. Additional informed consented was obtained from all patients for which identifying information is not included in this article.

Human and animal rights This article does not contain any studies with human or animal subjects performed by any of the authors. 


\section{References}

1. Amin VR (1989) Ultrasonic attenuation estimation for tissue characterization" Retrospective Theses and Dissertations. 17318. https://lib.dr.iastate.edu/rtd/17318

2. Tole NM (2005) Basic physics of ultrasonographic imaging. Harald Ostensen (ed) Diagnostic Imaging and Laboratory Technology Essential Health Technologies Health Technology and Pharmaceuticals WORLD HEALTH ORGANIZATION Geneva

3. Sassaroli E, O'Neill BE (2014) Modulation of the interstitial fluid pressure by high intensity focused ultrasound as a way to alter local fluid and solute movement: insights from a mathematical model. Phys Med Biol 59(22):6775-6795

4. Cardenas-Garcia J, Mayo PH, Folch E (2015) Ultrasonographic evaluation of the Pleura. Sage J. https://doi.org/10.1177/23739 97515610270

5. Hendee WR, O'Connor MK (2012) Radiation risks of medical imaging: separating fact from fantasy. Radiology 264:312-321

6. Trinavarat P, Riccabona M (2014) Potential of ultrasound in the pediatric chest. Eur J Radiol 83(9):1507-1518. https://doi. org/10.1016/j.ejrad.2014.04.011

7. Kim OH, Kim WS, Kim MJ, Jung JY, Suh JH (2000) US in the diagnosis of pediatric chest diseases. Radio Graphics 20:653-671

8. Coley BD (2005) Pediatric chest ultrasound. Radiol Clin North Am 43(2):405-418

9. Cox M, Soudack M, Podberesky DJ, Epelman M (2017) Pediatric chest ultrasound: a practical approach. Pediatr Radiol 47(9):10581068. https://doi.org/10.1007/s00247-017-3896-8

10. Goh Y, Kapur J (2016) Sonography of the pediatric chest. J Ultrasound Med 35(5):1067-1080. https://doi.org/10.7863/ultra .15 .06006

11. Sperandeo M, Varriale A, Sperandeo G, Bianco MR, Piattelli ML, Bizzarri M, Ghittoni G, Copetti M, Vendemiale G (2011) Characterization of the normal pulmonary surface and pneumonectomy space by reflected ultrasound. J Ultrasound 14(1):22-27. https:// doi.org/10.1016/j.jus.2011.01.004

12. Marchetti G, Arondi S, Baglivo F, Lonni S, Quadri F, Valsecchi A, Venturoli N, Ceruti P (2018) New insights in the use of pleural ultrasonography for diagnosis and treatment of pleural disease. Clin Respir J 12(6):1993-2005. https://doi.org/10.1111/crj.12907

13. Volpicelli G, Caramello V, Cardinale L, Mussa A, Bar F, Frascisco MF (2008) Detection of sonographic B-lines in patients with normal lung or radiographic alveolar consolidation. Med Sci Monit 14(3):122-128

14. Soldati G, Demi M, Inchingolo R, Smargiassi A, Demi L (2016) On the Physical Basis of Pulmonary Sonographic Interstitial Syndrome. J Ultrasound Med 35:2075-2086

15. Lichtenstein D, Mézière G, Biderman P, Gepner A, Barré O (1997) The comet-tail artefact. An ultrasound sign of alveolar-interstitial syndrome. Am J Respir Crit Care Med 156(5):1640-1646
16. Sperandeo M, Filabozzi P, Varriale A, Carnevale V, Piattelli ML, Sperandeo G, Brunetti E, Decuzzi M (2008) Role of thoracic ultrasound in the assessment of pleural and pulmonary diseases. $\mathrm{J}$ Ultrasound 11(2):39-46. https://doi.org/10.1016/j.jus.2008.02.001

17. Soni NJ, Franco R, Velez MI, Schnobrich D, Dancel R, Restrepo MI, Mayo PH (2015) Ultrasound in the diagnosis and management of pleural effusions. J Hosp Med 10(12):811-816. https:// doi.org/10.1002/jhm.2434Epub 2015 Jul 28

18. Trovato GM, Sperandeo M, Catalano D (2013) Thoracic ultrasound guidance for access to pleural, peritoneal, and pericardial space. Chest 144:1735-17356

19. Pereda MA, Chavez MA, Hooper-Miele CC, Gilman RH, Steinhoff MC, Ellington LE, Gross M, Price C, Tielsch JM, Checkley W (2015) Lung ultrasound for the diagnosis of pneumonia in children: a meta-analysis. Pediatrics 135(4):714-722. https://doi. org/10.1542/peds.2014-2833

20. Reali F, Sferrazza Papa GF, Carlucci P, Fracasso P, Di Marco F, Mandelli M, Soldi S, Riva E, Centanni S (2014) Can lung ultrasound replace chest radiography for the diagnosis of pneumonia in hospitalized children? Respiration 88(2):112-115. https://doi. org/10.1159/000362692

21. Sperandeo M, Tinti MG, Rea G (2017) Chest ultrasound versus chest X-rays for detecting pneumonia in children: Why compare them each other if together can improve the diagnosis? Eur J Radiol 93:291-292. https://doi.org/10.1016/j.ejrad.2017.05.038

22. Catalano D, Trovato G, Sperandeo M, Sacco MC (2014) Lung ultrasound in pediatric pneumonia. The persistent need of chest X-rays. Pediatr Pulmonol 49(6):617-618. https://doi.org/10.1002/ ppul.22941

23. Hoskins PR, Martin K, Thrush A (2010) Diagnostic ultrasound: physics and equipment. Cambridge University Press, Cambridge

24. Liu J, Liu Y, Wang HW, Li JY, Han T, Liang J, Yang CS, Xing M, Feng ZC (2013) Lung ultrasound for diagnosis of neonatal atelectasis. Zhonghua Er Ke Za Zhi 51(9):644-648

25. Kreuter M, Mathis G (2014) Emergency ultrasound of the chest. Respiration 87(2):89-97. https://doi.org/10.1159/000357685

26. Fulton C, Bratu I (2015) Occult pneumothoraces in ventilated pediatric trauma patients: a review. Can J Surg 58(3):177-180

27. Liu J (2014) Lung ultrasonography for the diagnosis of neonatal lung disease. J Matern Fetal Neonatal Med 27(8):856-861. https ://doi.org/10.3109/14767058.2013.844125

28. Riu B, Ruiz J, Mari A, Silva S (2013) Chest ultrasonography in pediatric critical care practice. Ann Fr Anesth Reanim 32(12):e219-e223. https://doi.org/10.1016/j.annfar.2013.10.007

Publisher's Note Springer Nature remains neutral with regard to jurisdictional claims in published maps and institutional affiliations. 